\title{
Electromagnetic Ion Cyclotron Waves in the High Altitude Cusp: Polar Observations
}

\author{
G. Le ${ }^{1,2}$, X. Blanco-Cano ${ }^{3}$, C. T. Russell ${ }^{1}$, X.-W. Zhou ${ }^{1}$, \\ F. Mozer ${ }^{4}$, K. J. Trattner ${ }^{5}$, S. A. Fuselier ${ }^{5}$ and B. J. Anderson ${ }^{6}$ \\ ${ }^{1}$ Institute of Geophysics and Planetary Physics, University of California, Los Angeles, California \\ ${ }^{2}$ Now at Laboratory for Extraterrestrial Physics, NASA Goddard Space Flight Center, Greenbelt, \\ Maryland \\ ${ }^{3}$ Instituto de Geofisica, UNAM, Ciudad Universitaria, Coyoacan, C.P. 04510, D.F., Mexico. \\ ${ }^{4}$ Space Sciences Laboratory, University of California, Berkeley, California \\ ${ }^{5}$ Lockheed-Martin Palo Alto Research Laboratory, Palo Alto, California \\ ${ }^{6}$ The Johns Hopkins University Applied Physics Laboratory, Laurel, Maryland
}




\begin{abstract}
High-resolution magnetic field data from the Polar Magnetic Field Experiment (MFE) show that narrow band waves at frequencies $\sim 0.2$ to $3 \mathrm{~Hz}$ are a permanent feature in the vicinity of the polar cusp. The waves have been found in the magnetosphere adjacent to the cusp (both poleward and equatorward of the cusp) and in the cusp itself. The occurrence of waves is coincident with depression of magnetic field strength associated with enhanced plasma density, indicating the entry of magnetosheath plasma into the cusp region. The wave frequencies are generally scaled by the local proton cyclotron frequency, and vary between 0.2 and 1.7 times local proton cyclotron frequency. This suggests that the waves are generated in the cusp region by the precipitating magnetosheath plasma. The properties of the waves are highly variable. The waves exhibit both lefthanded and right-handed polarization in the spacecraft frame. The propagation angles vary from nearly parallel to nearly perpendicular to the magnetic field. We find no correlation among wave frequency, propagation angle and polarization. Combined magnetic field and electric field data for the waves indicate that the energy flux of the waves is guided by the background magnetic field and points downward toward the ionosphere.
\end{abstract}




\section{Introduction}

The Polar spacecraft, in its highly elliptical orbit with an apogee of $9 R_{E}$ and an inclination angle of $86^{\circ}$ provides an excellent opportunity to study the polar cusp at high altitudes. When passing through the polar cusp region near apogee, the Polar spacecraft often enters a region with magnetosheath-like plasma, or the polar cusp, that can be readily identified by a sudden increase of the low-energy plasma density in the data from Hot Plasma Analyzer (Hydra) [Scudder et al., 1995] and Toroidal Imaging Mass-Angle Spectrograph (TIMAS) [Shelley et al., 1995] and an associated diamagnetic magnetic field depression in the magnetic field data (MFE) [Russell et al., 1995]. Zhou et al. $[1999,2000]$ reported the studies on the location and the solar wind control of polar cusp at high altitudes. In this paper, we report a study of the electromagnetic ion cyclotron waves in the polar cusp region as well as in the region adjacent to the cusp observed in the Polar magnetic field data.

In the high latitude region, electromagnetic ion cyclotron waves have been reported in the data from low-altitude polar orbiting spacecraft such as Injun 5 at altitudes less than $3000 \mathrm{~km}$ [Gurnett and Frank, 1972], S3-3 between altitudes 800 to $8000 \mathrm{~km}$ [Temerin and Lysak, 1984], ISIS 2 between altitudes 500 to $4000 \mathrm{~km}$ [Saito et al., 1987], and Freja between altitudes 1200 to $1750 \mathrm{~km}$ [Erlandson and Zanetti, 1998]. The waves were narrow-banded with frequencies between the local proton $\left(\mathrm{H}^{+}\right)$cyclotron frequency $(\mathrm{fcp})$ and the local $\mathrm{He}^{+}$particle cyclotron frequency ranging from 20 to $400 \mathrm{~Hz}$. They were found only in a narrow range of auroral latitudes in the pre-midnight sector in association with low-energy auroral electron precipitation. At mid altitudes, the electromagnetic ion cyclotron waves were observed in the polar cusp region at $\sim 4 \mathrm{R}_{\mathrm{E}}$ altitude by OGO 5 [Russell et al., 1971; Scarf et al., 1972; Fredricks and Russell, 1973] and at $\sim 2 \mathrm{R}_{\mathrm{E}}$ altitude by Viking [Erlandson et al., 1988]. The wave frequencies at higher altitudes were observed in the range between the local $\mathrm{H}^{+}$ 
cyclotron frequency and alpha particle $\mathrm{H}^{+}$cyclotron frequency, $4-7 \mathrm{~Hz}$ at $\mathrm{OGO} 5$ and 18 to $27 \mathrm{~Hz}$ at Viking. The anisotropies in the electron and ion distributions were suggested as the energy sources for the waves in the cusp [Erlandson et al., 1988].

A survey of high-resolution magnetic field data from the Polar spacecraft reveals that the electromagnetic ion cyclotron waves are a permanent feature in the polar cusp. The Polar data extended the observations of cusp electromagnetic ion cyclotron waves to high altitudes $\sim 5$ to $9 R_{E}$. In this paper, we summarize the characteristics of ion cyclotron waves in the high altitude cusp region as observed in the Polar magnetic field data. We focus on observational aspects; the generation mechanism and plasma instabilities are a topic of future study. 


\section{Polar Magnetic Field Data}

Russell et al. [1995] described the Magnetic Field Experiment (MFE) on the Polar spacecraft. The magnetic field data were transmitted to Earth at a constant rate of $8.33 \mathrm{~Hz}$ or $120 \mathrm{msec}$ per sample, corresponding to a Nyquist frequency of $4.17 \mathrm{~Hz}$. In this study, we have surveyed this highresolution magnetic field data during Polar's passages of the cusp region in high altitude northem hemisphere during the time period from April 1 to December 31, 1996. There are totally 212 cusp passages during this time period identified by Zhou et al. [1999] using combined Polar magnetic field data, Hydra key parameter data and TIMAS key parameter data. These cusp entries occur at altitudes from 4.8 to $8.8 \mathrm{R}_{\mathrm{E}}$. The magnetic field strength at these cusp crossings ranges from 36 to $467 \mathrm{nT}$. Among the 212 cusp passages, we have found evidence of the existence of waves in 197 passages, which occur as narrow-band waves in the frequency range from $\sim 0.2 \mathrm{~Hz}$ up to $\sim 4 \mathrm{~Hz}$. Waves with higher frequencies may still present, but can not be resolved by Polar magnetic field data at its normal $8.33 \mathrm{~Hz}$ data rate.

\section{Wave Frequencies}

Before we present examples of these waves, we first show in Figure 1 the frequencies of these waves as a function of local magnetic field strength from the 197 cusp passages with waves. Note that the waves observed in the same cusp crossing may occur at markedly different frequencies. Multiple wave frequencies may be included from selected wave packets in each cusp crossing, thus, the data points in Figure 1 are not independent to each other and one may not attempt to obtain the information of occurrence rate of wave frequencies from them. It is only intended to show the range of frequency variance. Figure 1 clearly shows that the wave frequencies are generally controlled by the local magnetic field strength. The wave frequencies normalized by the local proton cyclotron 
frequencies are in the range of 0.2 to 1.7 . This observation strongly suggests that these waves are generated locally in the cusp region. Although waves in the same frequency band occur in the magnetosheath [Anderson and Fuselier, 1993; Schwartz et al., 1996], it is very unlikely that waves in the cusp have propagated from the magnetosheath and then exhibit the control by the local magnetic field strength shown in this plot.

The vertical bars in Figure 1 indicate the average local magnetic field $B$ for the 15 cusp crossings where no evidence of the existence of waves is found. It is evident that the majority of the waves tend to be observed in the cusp crossings with smaller magnetic field strength. For all 181 cusp passages with $\mathrm{B}<250 \mathrm{nT}$, we see clear evidence of the existence of the waves. The crossings without the waves tend to have large magnetic field. The local magnetic field strength is greater than $\sim 250$ $\mathrm{nT}$ for all the 15 crossings without the waves ( 15 out of 31 passages with $\mathrm{B}>250 \mathrm{nT}$ ). Thus, it is possible that there are still waves present but at higher frequencies beyond the instrument bandwidth for Polar MFE.

Analysis of the wave properties shows that they are variable. The propagation angles vary in the whole range, from nearly parallel to nearly perpendicular to the background magnetic field. The waves exhibit both left-handed and right-handed polarization in the spacecraft frame with a broad range of ellipticity (from nearly linear to nearly circular polarization). We do not find any correlation among wave frequency, propagation angle, and polarization. It is possible that instabilities are at work generating both left-handed and right-handed wave modes in the cusp region. Even for waves occurring at frequencies above the local proton cyclotron frequency, both left-handed and righthanded waves are observed in the spacecraft frame. In the plasma dispersion relation, the lefthanded/Alfven waves cannot propagate either in the direction perpendicular to the magnetic field or 
at frequencies above the ion cyclotron frequency. Thus the waves observed above the proton cyclotron frequency should be in the right-handed/magnetosonic mode. Why some of the waves exhibit left-handed polarization above the ion cyclotron frequency is still a puzzle. The righthanded/magnetosonic mode above the proton cyclotron frequency has phase velocity above Alfven velocity. For typical conditions in the cusp at Polar altitudes, Alfven velocity $(\sim 1000 \mathrm{~km} / \mathrm{s})$ is much greater than the plasma flow velocity $(\sim 100 \mathrm{~km} / \mathrm{s})$, thus Doppler shift cannot reverse the wave polarization from right-handed in the plasma rest frame to left-handed in the spacecraft frame. Under some extreme conditions with highly depressed magnetic field and enhanced plasma density, or with presence of high percentage of heavy ions in the cusp, Alfven velocity may become comparable to plasma flow velocity and Doppler shifting maybe involved in the observations of left-handed waves in the spacecraft frame. 


\section{Examples of Waves in the Cusp}

The May 24, 1996 Cusp Crossing. Figure 2 shows an overview of the high altitude polar cusp crossing on May 24, 1996 (from 0030 to 0440 UT), where the narrow-band waves are observed throughout the passage. During this crossing, the Polar orbit plane was roughly in the noon-midnight meridian plane moving towards high latitudes. In Figure 2, the top panel shows the magnetic field data and bottom panel shows Hydra electron density and average energy key parameters. The spacecraft crossed the cusp region from the dayside magnetosphere $(\mathrm{Bx}<0)$ to the tail lobe $(\mathrm{Bx}>0)$. The signatures for the polar cusp are characterized by a depression of the magnetic field strength and an enhancement of low energy plasma density associated with the precipitation of the magnetosheath plasma into the cusp region $(\sim 0052$ to 0140 UT). When the spacecraft was just equatorward of the cusp and after the spacecraft went into the lobe region poleward of the cusp, there were multiple encounters of magnetosheath-like cusp plasma pulses with a magnetic field depression lasting from less than one minutes to a few minutes, probably due to the motion of the polar cusp as well as the unsteady nature of magnetosheath plasma precipitations into the cusp region.

The existence of the waves in the cusp region is evident in the magnetic field data in Figure 2. We find that the wave properties vary in the same cusp crossing, which is a very common phenomenon in the data surveyed. To illustrate the details, we select several short intervals for a close examination of wave properties. Figure 3 shows an interval of waves observed inside the cusp region from 0122:00 to 0123:20 UT. The narrow band waves at $\sim 1.2 \mathrm{~Hz}$ with peak-to-peak amplitude $\leq 4 \mathrm{nT}$ were found in the region with a $10 \mathrm{nT}$ depression of the magnetic field, as shown in the top panel of Figure 3. The wave frequency weighted by spectral power is about $0.96 \mathrm{fcp}$, only slightly lower the local proton cyclotron frequency. The waves, while having similar frequency in 
this short interval, exhibit very different polarization and propagation directions. In the lower panels of Figure 3, we present hodograms of wave packet labeled a, $b$ and $c$ in the top panel, where $\delta \mathrm{B}_{\mathfrak{l}}, \delta \mathrm{B}_{\text {, }}$ and $\delta \mathrm{B}_{\mathrm{K}}$ correspond to the wave magnetic field in the directions of their maximum (I), intermediate $(\mathrm{J})$ and minimum $(\mathrm{K})$ variance directions, respectively. The arrows labeled $\mathbf{B}$ are the projection of the local background magnetic field with magnitude $\sim 82 \mathrm{nT}$. The planes of oscillation have changed markedly for these wave packets. For wave packet a, the angle between the background magnetic field and the wave $\mathrm{k}$ vector $\left(\theta_{\mathrm{Bk}}\right)$ is $57^{\circ}$ and the wave is clearly right-handed polarized with an ellipticity of 0.64 . For wave packet $b$, the polarization changes to left-handed although the ellipticity $(0.68)$ and $\theta_{\mathrm{Bk}}\left(56^{\circ}\right)$ keep roughly the same. For the next wave packet $\mathrm{c}$, the background magnetic field lies roughly in the plane of oscillation so that the wave propagates at very oblique angle, $86^{\circ}$ from the local magnetic field.

The frequency of the wave is also quite variable for the same cusp crossing. Figure 4 shows another interval of the May 24, 1996 cusp crossing with waves occurring at much lower frequency than those in Figure 3. The top panel of Figure 4 contains magnetic field data and is plotted in the same time scale as in the top panel of Figure 3. The power spectrum for the entire interval is shown in the lower panel. The power spectrum contains multiple peaks at frequencies lower than local proton cyclotron. The frequency of the peak with maximum power is $0.60 \mathrm{~Hz}$, or $0.44 \mathrm{fcp}$.

In our survey, the waves are most frequently observed within and adjacent to the field depression in the cusp region. The May 24, 1996 cusp crossing provides a good example, where the waves are also observed in the short pulses of field depression region both equatorward and poleward of the cusp region. Figure 5 shows the magnetic field data and their power spectra in four 
of the field depression regions in the lobe on May 24, 1996. The waves are seen inside the field depression region in Figure 5b-c and both inside and near the edge of the field depression region in Figure $6 \mathrm{a}$ with variable frequencies. The average frequencies of the spectral peaks weighted by the power in these field depression regions are (a) $1.48 \mathrm{~Hz}$ or $0.90 \mathrm{fcp}$, (b) $0.49 \mathrm{~Hz}$ or $0.32 \mathrm{fcp}$, (c) 1.36 $\mathrm{Hz}$ or $0.84 \mathrm{fcp}$, and (d) $1.23 \mathrm{~Hz}$ or $0.78 \mathrm{fcp}$, respectively. In all the cases, the association of the waves and the magnetic field depression is very clear. The depression of the magnetic field is required by maintaining the pressure balance with the precipitation of magnetosheath plasma. The occurrence of the waves in association with the magnetic field depression suggests that the instabilities are most likely caused by entering magnetosheath plasma.

The September 10,1996 Cusp Crossing. We now show another example of cusp crossing on September 10, 1996. Figure 6 shows the overview of the magnetic field data and Hydra plasma data for the interval of interest. Polar orbit plane was roughly in the noon-midnight meridian plane. The spacecraft moved from high latitudes to low latitudes, i.e., from the tail lobe $(\mathrm{Bx}>0)$ to the cusp region, and then into the dayside magnetosphere $(\mathrm{Bx}<0)$. The crossing of the polar cusp occurred from $\sim 0700$ to 0915 UT and there were a few short excursions of magnetosheath-like cusp plasma associated with the magnetic field depression before and after the cusp crossing. Again, the narrow band waves are observed in the cusp region and in all intervals with magnetosheath-like cusp plasma pulses and magnetic field depression. In Figure 7 we present three short wave intervals in the field depression region poleward of the polar cusp ( $a$ and $b$ ) and in the cusp itself (c). It is also evident that the waves have variable frequencies even within a short period of time

In some intervals during this cusp crossing, we have seen co-existence of multiple narrow band waves at different frequencies in the same time. This type of waves with multiple spectral 
peaks is found in 17 out of 197 cusp passages in the time period surveyed. Figure 8 shows the wave magnetic field data and the power spectrum from 0724:28 to 0725:18 UT on September 10, 1996. The magnetic field clearly shows the existence of multiple frequencies. The two narrow band spectral peaks occurred at $0.49 \mathrm{~Hz}(0.51 \mathrm{fcp})$ and $0.91 \mathrm{~Hz}(0.96 \mathrm{fcp})$. The lower frequency peak occurs very close to the local alpha particle $\left(\mathrm{He}^{++)}\right.$cyclotron frequency and the higher frequency peak close to the local proton cyclotron frequency. The wave analysis shows that the lower frequency wave propagates at $4^{\circ}$ from the background magnetic field and is left-handed polarized with an ellipticity of -0.88 . The higher frequency wave propagates at $22^{\circ}$ from the background magnetic field and is right-handed polarized with an ellipticity of 0.30 . Dual spectral peaks associated with proton and alpha particle cyclotron frequencies have been observed previously in the magnetosheath, but the polarization of the waves is just opposite to the case in this cusp crossing [Anderson and Fuselier, 1993]. This difference in polarization may be additional evidence that waves in the cusp are not those propagating into the cusp from the magnetosheath.

The April 21, 1996 Cusp Crossing. The April 21, 1996 crossing is one of those when the polar cusp was crossed at lowest altitude in the Polar data surveyed. During this crossing, the Polar orbit plane was also near the noon-midnight meridian and the spacecraft crossed the cusp region from high latitudes to low latitudes in the dayside magnetosphere. Figure 9 shows the magnetic field data and Hydra plasma data around the cusp crossing, indicating the polar cusp was seen from 1840 to 1910 UT. The altitude of the spacecraft at cusp crossing is $\sim 5 R_{E}$, much lower than those in the previous two examples as well as in the majority of the cusp crossings. The waves are found in the higher latitude part of the cusp region. A wave interval is shown in Figure 10 for period from 1844:00 to 1846:30 UT. The presence of waves is evident in the magnetic field data in the top panel of Figure 
10. The power spectra are shown in the bottom panels of Figure 10 for periods (a) 1844:13-1844:22 UT, (b) 1844:48 - 1844:57, and (c) 1845:33-1845:43. In the power spectrum for period a, the spectral peak occurs at $1.59 \mathrm{~Hz}$, or $0.24 \mathrm{fcp}$. The wave propagates at $7^{\circ}$ from the background magnetic field and is right-handed polarized with an ellipticity of 0.41 . There is also a possible spectral peak at frequency greater than $3 \mathrm{~Hz}$, which cannot be fully resolved by Polar MFE at normal data rate. For period $b$, the spectral peak occurs at $2.05 \mathrm{~Hz}$, or $0.31 \mathrm{fcp}$. The wave propagates at $2^{\circ}$ from the background magnetic field and is left-handed polarized with an ellipticity of 0.53 . For period $c$, the spectral peak occurs at $2.56 \mathrm{~Hz}$, or $0.40 \mathrm{fcp}$. The wave propagates at $65^{\circ}$ from the background magnetic field data and is left-handed polarized with an ellipticity of -0.20 . Again, the waves occur at different frequencies and exhibit a wide range of propagating angles and senses of polarization. 


\section{Poynting Flux of Waves}

We have used the data from both the Polar MFE and Electric Fields Investigation (MFI) [Harvey et al., 1995] to calculate the Poynting flux of the waves. We examined the Poynting flux of the waves from three cusp crossings in 1997 (they are not included in our statistical survey for 1996 data). The electric field data are available at 0.05 or $0.025 \mathrm{~s}$ time resolution, depending on the telemetry rate. We averaged them down to the same time resolution of the magnetic field data to calculate the Poynting flux.

Figure 11 shows a wave interval from the April 11, 1997 cusp crossing. The electric field data from all three axes are available for this interval. In Figure 11, we present high-pass filtered magnetic field $(\delta \mathbf{B})$ and electric field data $(\delta \mathbf{E})$, calculated wave Poynting flux $\mathbf{S}=\delta \mathbf{E} \times \delta \mathbf{B}$ in the direction parallel and perpendicular to the background magnetic field, as well as $\delta \mathrm{E} / \delta \mathrm{B}$ (an estimate of wave phase velocity) for two wave packets observed in the cusp. It is clear that the wave Poynting flux is largely guided by the background magnetic field and pointing downward towards the ionosphere. For both the wave packets, the phase velocity is in the order of $800 \mathrm{~km} / \mathrm{s}$, which is in the same order of Alfven velocity $(\sim 980 \mathrm{~km} / \mathrm{s})$ estimated from local magnetic field strength ( $100 \mathrm{nT})$ and plasma density $\left(\sim 5 \mathrm{~cm}^{-3}\right)$. The weighted wave frequency is $1.17 \mathrm{fcp}$ and the wave is left-handed polarized in the spacecraft with ellipticity of 0.58 .

The next two cusp crossings we examined occurred on July 3 and July 31, 1997. During this period of time, the electric field data from one axis is perturbed due to the boom going into the spacecraft shadow twice every spacecraft spin $(6 \mathrm{~s})$. Thus this component of the electric field data was set to zero and the electric field data from two axes are used in the calculation. This will mainly cause the GSM Y component of the electric field data $(\delta E y)$ to be underestimated. But the data 
should still be able to provide us information on the direction of the Poynting flux as long as the GSM Y component of the magnetic wave is strong since $\delta \mathrm{E} \cdot \delta \mathrm{B}=0$. The waves we examined for July 3 and July 31, 1997 cusp crossings satisfy this condition. Figure 12 shows the wave interval during July 31, 1997 cusp crossing, including hi-pass filtered magnetic field and electric field data and calculated Poynting flux parallel and perpendicular to the background magnetic field data. In this case, the wave Poynting flux is also largely in the direction of the background magnetic field and pointing downward toward the ionosphere. For this example, the weighted frequency of the wave packet starting at $0636: 40 \mathrm{UT}$ is $1.10 \mathrm{fcp}$, and the wave is nearly linearly polarization with ellipticity of 0.11 . The Poynting flux for waves in July 3, 1997 cusp crossing is also largely in the direction of the background magnetic field and pointing downward toward the ionosphere (not shown). 


\section{Summary}

In a survey of high-resolution magnetic field data from the Polar Magnetic Field Experiment (MFE) in the cusp region, we find that narrow band waves at frequencies $\sim 0.2$ to $3 \mathrm{~Hz}$ are a permanent feature in the vicinity of the polar cusp. The waves have been found in the magnetosphere adjacent to the cusp (both poleward and equatorward of the cusp) and in the cusp itself. The occurrences of waves are coincident with the depression of magnetic field strength associated with enhanced plasma density, indicating the entry of magnetosheath plasma into the cusp region. The wave frequencies are generally scaled by the local proton cyclotron frequency, and vary between 0.2 and 1.7 times local proton cyclotron frequency. This suggests that these waves are generated in the cusp region by instabilities associated with precipitating magnetosheath plasma. Combined magnetic field and electric field data for the waves indicate that the energy flux of the waves is guided by the background magnetic field and points downward toward the ionosphere.

The properties of the waves in the cusp are highly variable. The waves exhibit both lefthanded and right-handed polarization in the spacecraft frame with a board range of ellipticity. The propagating angles vary from nearly parallel propagation to nearly perpendicular propagation. We do

not find any correlation among wave frequency, propagation angle and polarization. It is very likely different instabilities are at work and both wave modes (Alfven and magnetosonic modes) are present in the cusp region. Since the left-handed/Alfven waves cannot propagate either at the direction perpendicular to the magnetic field or at the frequency above the ion cyclotron frequency, the waves observed above the proton cyclotron frequency should be in right-handed/magnetosonic mode. However, this is not the case in the observation. We found both left-handed and right-handed waves above local proton cyclotron frequency. Since the plasma flow velocity in the cusp is an order 
of magnitude smaller than typical Alfven velocity at Polar altitude, it is unlikely that Doppler shift would be able to reverse the wave polarization under normal cusp conditions. It is only possible when Alfven velocity becomes comparable to the flow velocity in the cusp under conditions of extremely high plasma density, low magnetic field, and high percentage of heavy ions. This issue remains to be a puzzle.

The identification of wave modes and generation mechanisms are topics of our future study. The polar cusp is an important region to study the plasma instabilities and wave-particle interactions. Recent observations of plasma in the polar cusp have traced their origins to the magnetosheath [Nishida et al., 1993; Kremer et al., 1995], the magnetospheric boundary layer [Woch and Lundin, 1991] and the ionosphere [Thelin et al., 1990; Giles et al., 1994]. Various plasma instabilities have been identified in the cusp region, including those associated with temperature anisotropy [Andre et al., 1988], particle beam and ion loss cone [Koskinen et al., 1988], and velocity shears [Lakhina, 1990]. In our follow-up study, we will examine the plasma distributions measured by the POLAR Toroidal Imaging Mass-Angle Spectrograph (TIMAS) instrument and perform a kinetic dispersion analysis using observed ion characteristics to understand which instabilities are generating the wave. Our observations presented above suggest that a search for local plasma instabilities is reasonable. In our initial survey of Polar TIMAS data, we found two types of plasma distributions associated with the waves: (1) counterstreaming ions with entered magnetosheath ions moving downward towards the ionosphere and mirrored ions upward away from the ionosphere; (2) uni-directional magnetosheath ions moving towards the ionosphere. In both cases, the distribution function of entered magnetosheath ions shows temperature anisotropy with $T_{\perp}$ greater than $T_{1 /}$. For a given temperature anisotropy above 1 , the plasma tends toward instability as the plasma beta is increased. 
Thus, the depression of the magnetic field (diamagnetic effects) in the cusp effectively increases the plasma beta and may cause the plasma to be unstable. Our future work will concentrate on the local instabilities associated with features in plasma distribution functions. 
Acknowledgements. We wish to thank Jack Scudder and CDAWeb for providing Polar Hydra key parameter data. The work at UCLA was supported by the National Aeronautics and Space Administration (NASA) under research grant NAG5-7721. X. Blanco-Cano was supported by CONACYT grant J27852E. F. Mozer was supported by NASA under research grant 19982924. S. Fuselier and K. J. Trattner were supported by NASA under research grant NAS5-30302. 


\section{References}

Anderson, B. J., and S. A. Fuselier, Magnetic pulsation from 0.1 to $4.0 \mathrm{~Hz}$ and associated plasma properties in the Earth's subsolar magnetosheath and plasma depletion layer, J. Geophys. Res., 98, 1461, 1993.

Andre, M., G. B. Crew, W. K. Peterson, A. M. Persoon, C. J. Pollock, and M. J. Engebretson, Ion heating by broadband low-frequency waves in the cusp/cleft, J. Geophys. Res., 95, 20,809, 1990.

Erlandson, R. E., and L. J. Zanetti, A statistical study of auroral electromagnetic ion cyclotron waves, J. Geophys. Res., 103, 4627, 1998.

Erlandson, R. E., L. J. Zanetti, T. A. Potemra, M. Andre, and L. Matson, Observation of electromagnetic ion cyclotron waves and hot plasma in the polar cusp, Geophys. Res. Lett., $15,421,1988$.

Fredricks, R. W., and C. T. Russell, Ion cyclotron waves observed in the polar cusp, J. Geophys. Res., 78, 2917, 1973.

Giles, B. L., C. R. Chappell, T. E. Moore, R. H. Comfort, and J. H. Waite, Jr., Statistical survey of pitch angle distributions in core $(0-50 \mathrm{eV})$ ions from Dynamics Explorer 1: outflow in the auroral zone, polar cap, and cusp, J. Geophys. Res., 99, 17,483, 1994.

Gurnett, D. A., and L. A. Frank, ELF noise bands associated with auroral electron precipitation, $J$. Geophys. Res., 77, 3411, 1972.

Harvey, P., F. S. Mozer, D. Pankow, J. Wygant, N. C. Maynard, H. Singer, W. Sullivan, P. B. Anderson, R. Pfaff, T. Aggson, A. Pedersen, C. G. Falthammar, and P. Tanskannen, The eEectric Field Instrument on the Polar satellite, Space Sci. Rev., 7I, 583, 1995. 
Koskinen, H. E. J., G. Gustafsson, M. Andre, and R. Lundin, Viking observations of wave-particle interactions and ion wave instabilities in the high-latitude magnetosphere, Computer Physics Communications, 49, 75, 1988.

Kremser, G., J. Woch, K. Mursula, P. Tanskanen, B. Wilken, and R. Lundin, Origin of energetic ions in the polar cusp inferred from ion composition measurements by the Viking satellite, Annales Geophysicae, 13, 595, 1995.

Lakhina, G. S., Generation of ULF waves in the polar cusp region by velocity shear-driven kinetic Alfven modes, Astrophysics and Space Science, 165, 153, 1990.

Nishida, A., T. Mukai, H. Hayakawa, N. Kaya, and M. Fujimoto, Precipitation of solar wind like ions in the polar cap during northward interplanetary magnetic field, J. Geophys. Res. , 98, $11,449,1993$.

Russell, C. T., C. R. Chappell, M. D. Montgomery, M. Neugebauer, and F. L. Scarf, Ogo 5 observations of the polar cusp on November 1, 1968, J. Geophys. Res., 76, 6743, 1971.

Russell, C. T., R. C. Snare, J. D. Means, D. Pierce, D. Dearborn, M. Larson, G. Barr, and G. Le, The GGS/Polar magnetic fields investigation, Space Sci. Rev., 71, 563, 1995.

Saito, H., T. Yoshino, and N. Sato, Narrow-banded ELF emissions over the southern polar region, Planet. Space Sci., 35, 745, 1987.

Scarf, F. L., R. W. Fredricks, I. M. Green, and C. T. Russell, Plasma waves in the dayside polar cusp, 1, Magnetospheric observations, J. Geophys. Res., 77, 2274, 1972.

Schwartz, S. J., D. Burgess, J. J. Moses, Low-frequency waves in the Earth's magnetosheath: Present status, Ann. Geophysicae, 14, 1134, 1996.

Scudder, J., et al., HYDRA - A 3-dimensional electron and ion hot plasma instrument for the Polar 
spacecraft of the GGS mission, Space Sci. Rev., 71, 459, 1995.

Shelley, E. G., et al., The Toroidal Imaging Mass-Angle Spectrograph (TIMAS) for the Polar mission, Space Sci. Rev., 71, 497, 1995.

Temerin, M., and R. L. Lysak, Electromagnetic ion cyclotron mode (ELF) waves generated by auroral electron precipitation, J. Geophys. Res., 89, 2849, 1984.

Thelin, B., B. Aparicio, and R. Lundin, Observations of upflowing ionospheric ions in the mid-altitude cusp/cleft region with the Viking satellite, J. Geophys. Res., 95, 5931, 1990.

Woch, J., and R. Lundin, Temporal magnetosheath plasma injection observed with Viking: a case study, Annales Geophysicae, 9, 133, 1991.

Zhou, X.-W., C. T. Russell, G. Le, S. A. Fuselier, and J. D. Scudder, The polar cusp and its dependence on dipole tilt, Geophys. Res. Lett., 26, 429, 1999.

Zhou, X.-W., C. T. Russell, G. Le, S. A. Fuselier, and J. D. Scudder, Solar wind control of the polar cusp at high altitude, J. Geophys. Res., 105, 245, 2000. 


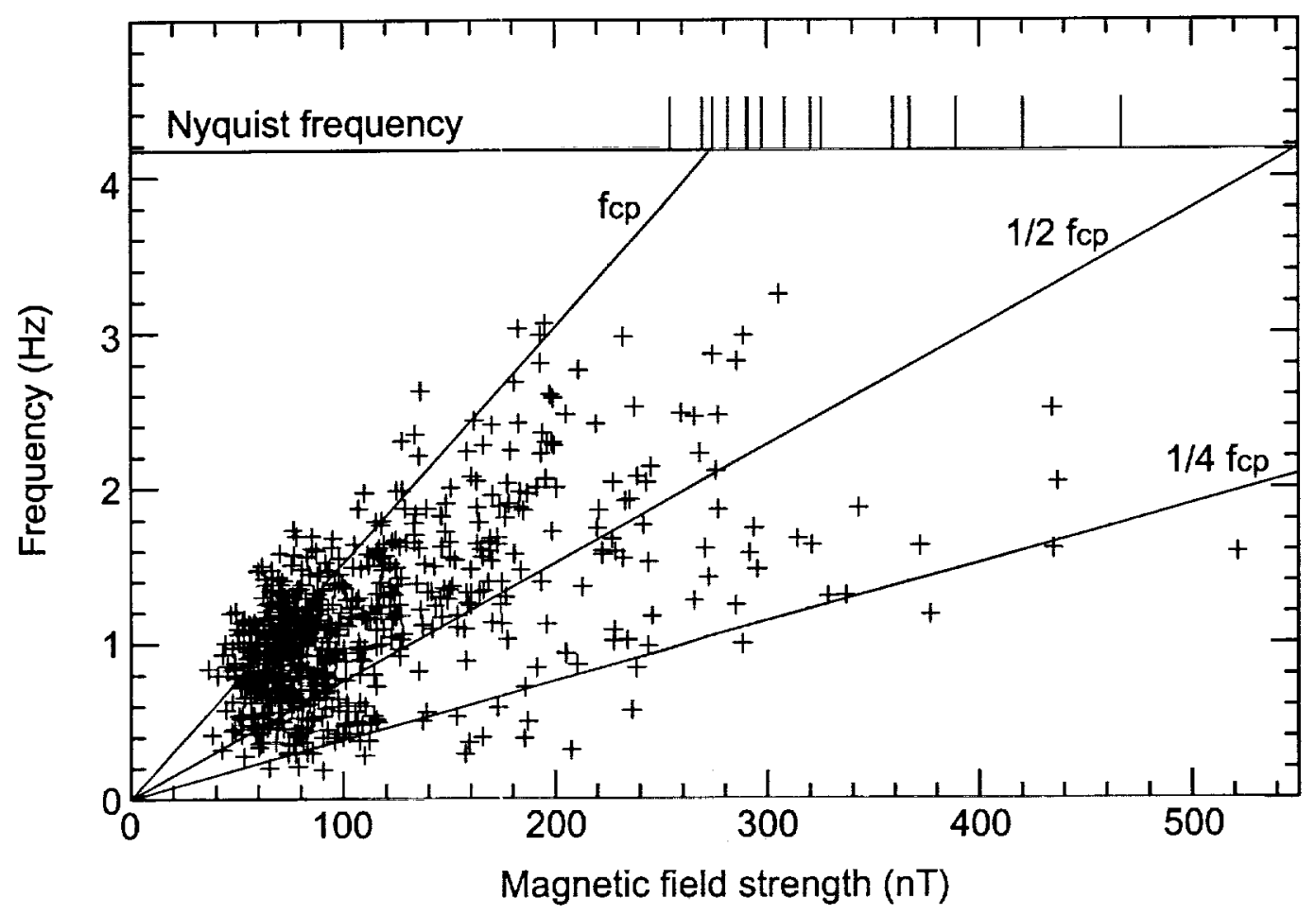

Figure 1. Frequencies of the waves versus the strength of the local magnetic field from the 197 cusp crossings. The vertical bars indicate the average magnetic field strength for the 15 cusp crossings where no evidence of the existence of waves is found. 

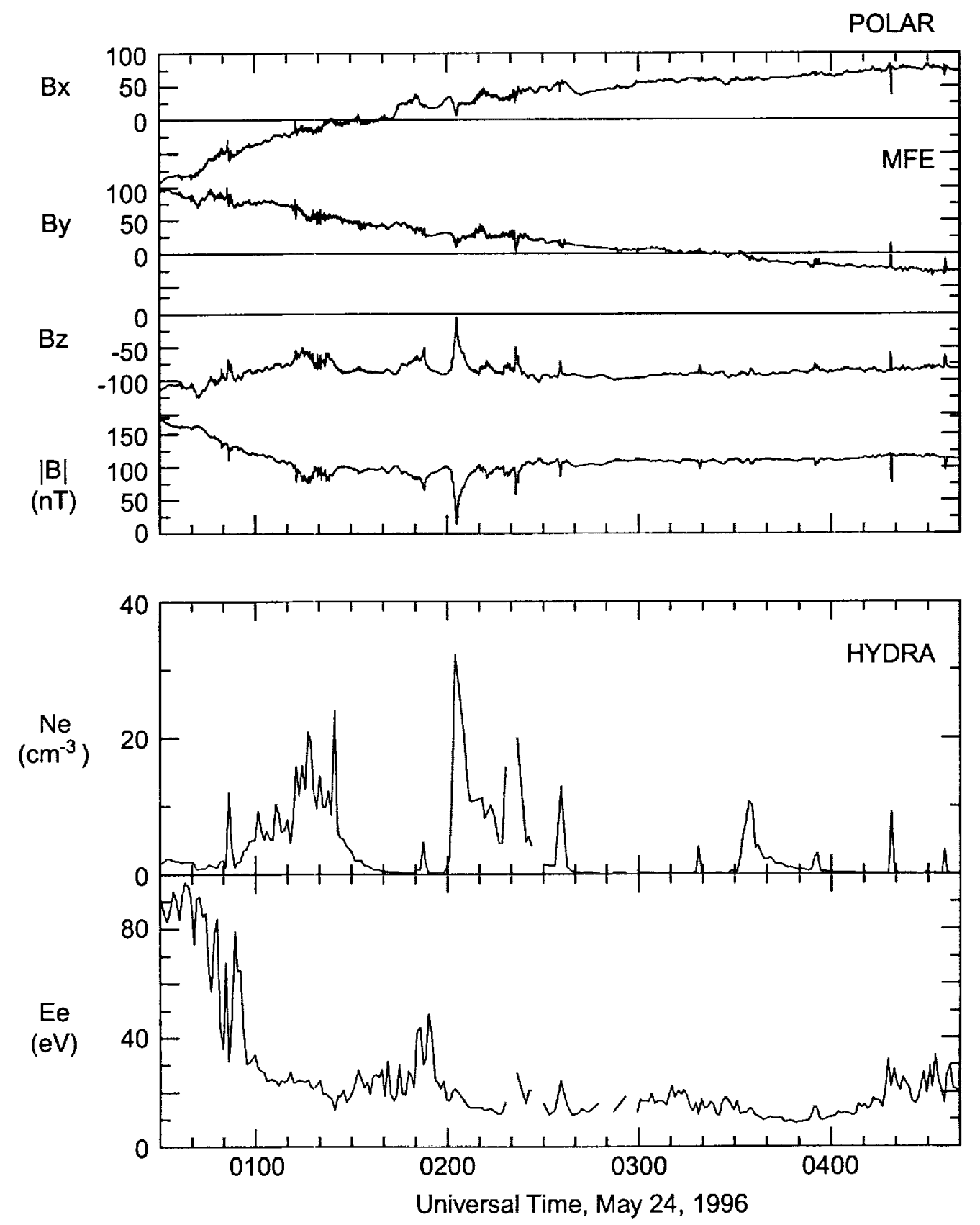

Figure 2. An overview of Polar observations of the cusp region on May 24, 1996. The top panel shows the magnetic field data in SM coordinates. The bottom panel shows Polar Hydra electron density and average energy key parameters. 

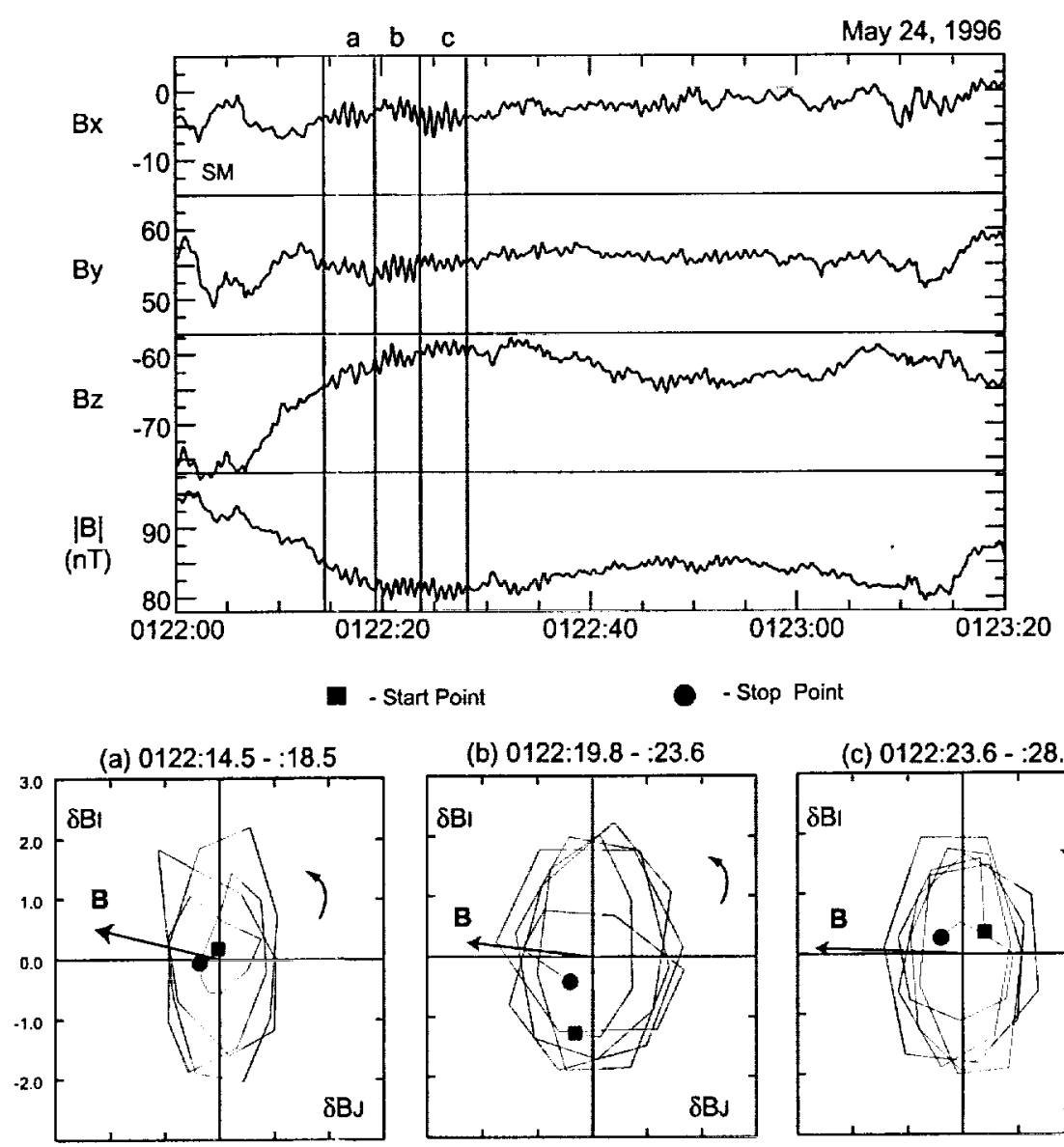

(c) $0122: 23.6-: 28.1$
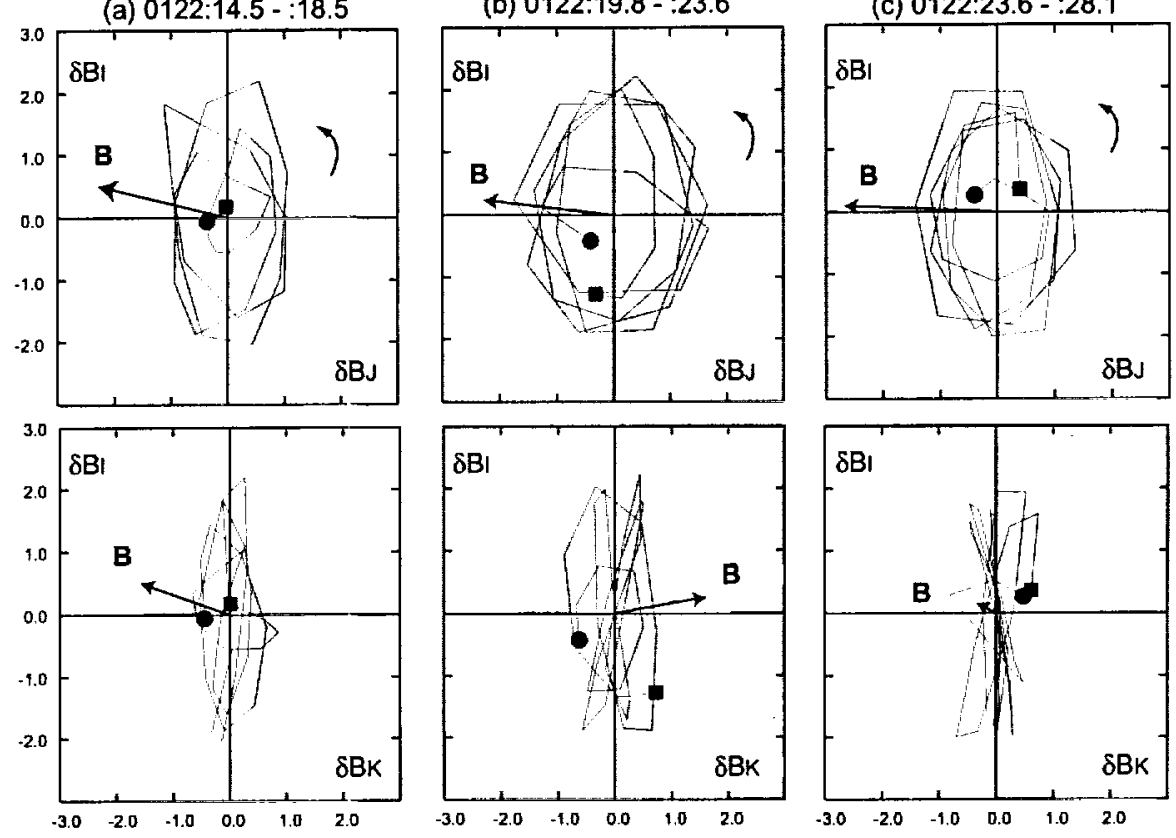

Figure 4

Figure 3. A wave interval observed inside the cusp region on May 24, 1996. The lower panels show hodograms wave magnetic field in the directions of their maximum (I), intermediate $(\mathrm{J})$ and minimum $(\mathrm{K})$ variances for three wave packets labeled $a, b$ and $c$ in the top panel. The arrows labeled $\mathrm{B}$ are the projection of the local background magnetic field of $82 \mathrm{nT}$ on the planes. 

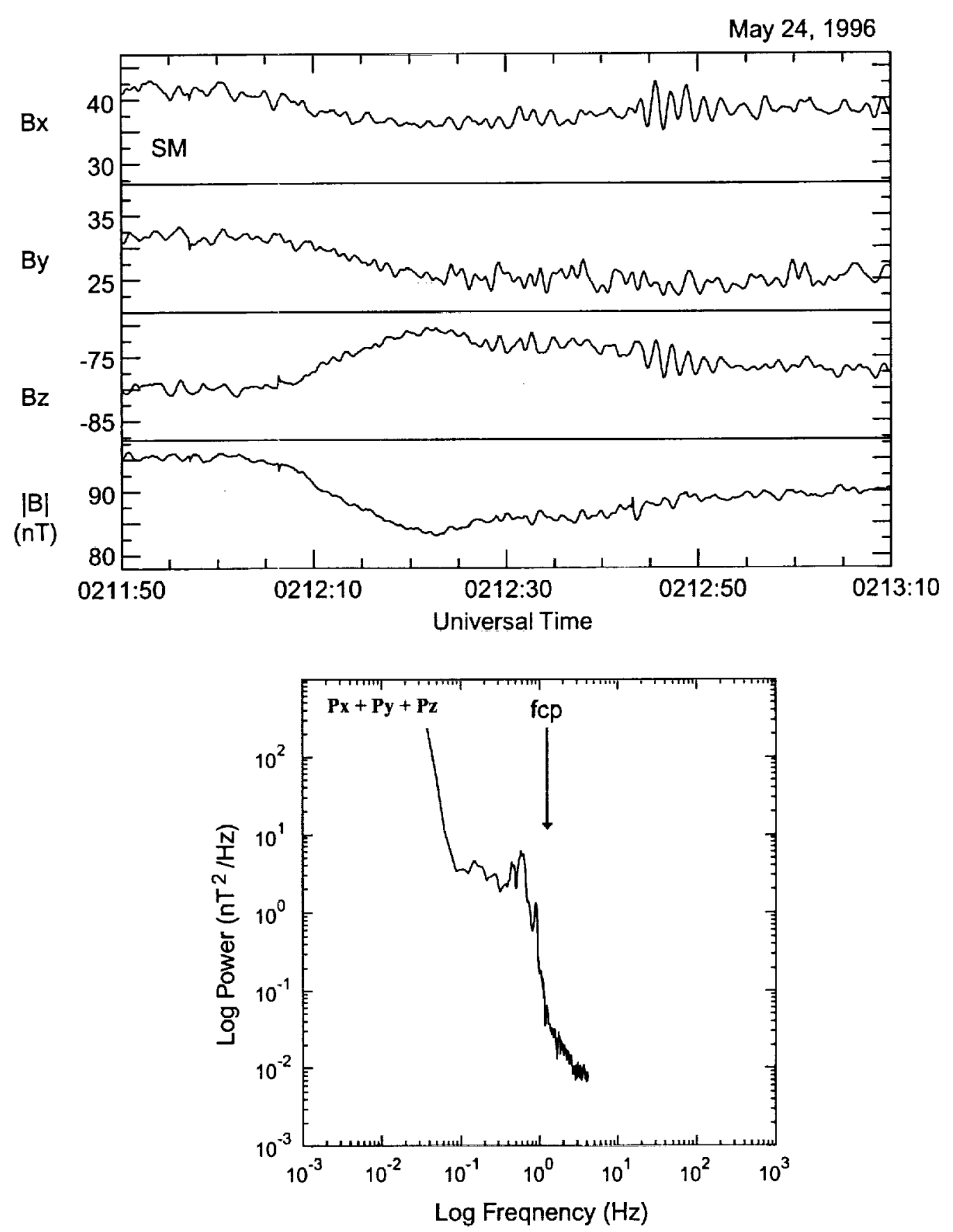

Figure 4. An example of the waves and their power spectrum on May 24, 1996. The frequency of the peak with maximum power is $0.60 \mathrm{~Hz}$, or $0.44 \mathrm{fcp}$. 

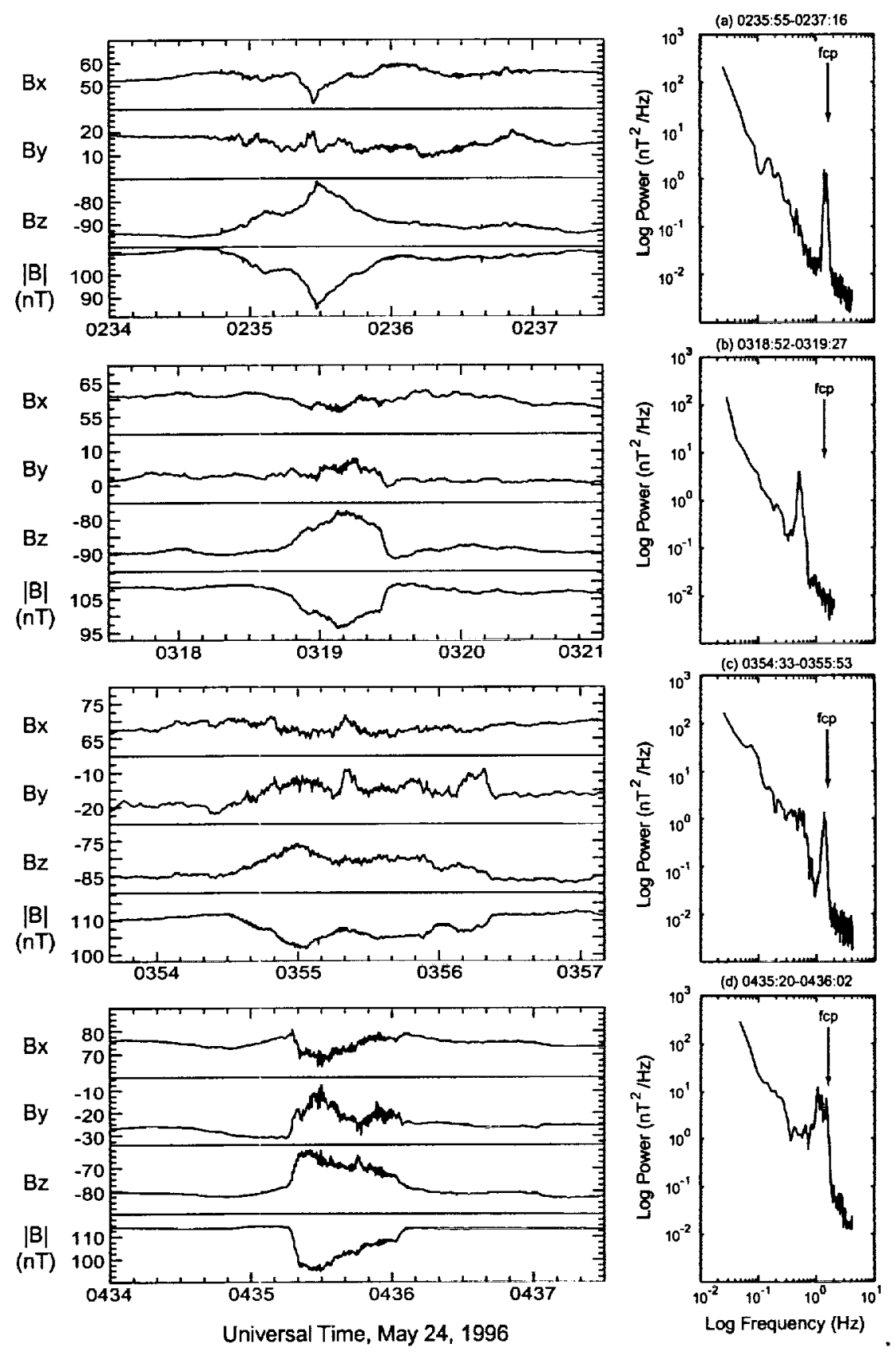

Figure 5. Examples of the waves and their power spectra observed within and adjacent to the field depression in the cusp region on May 24, 1996. 

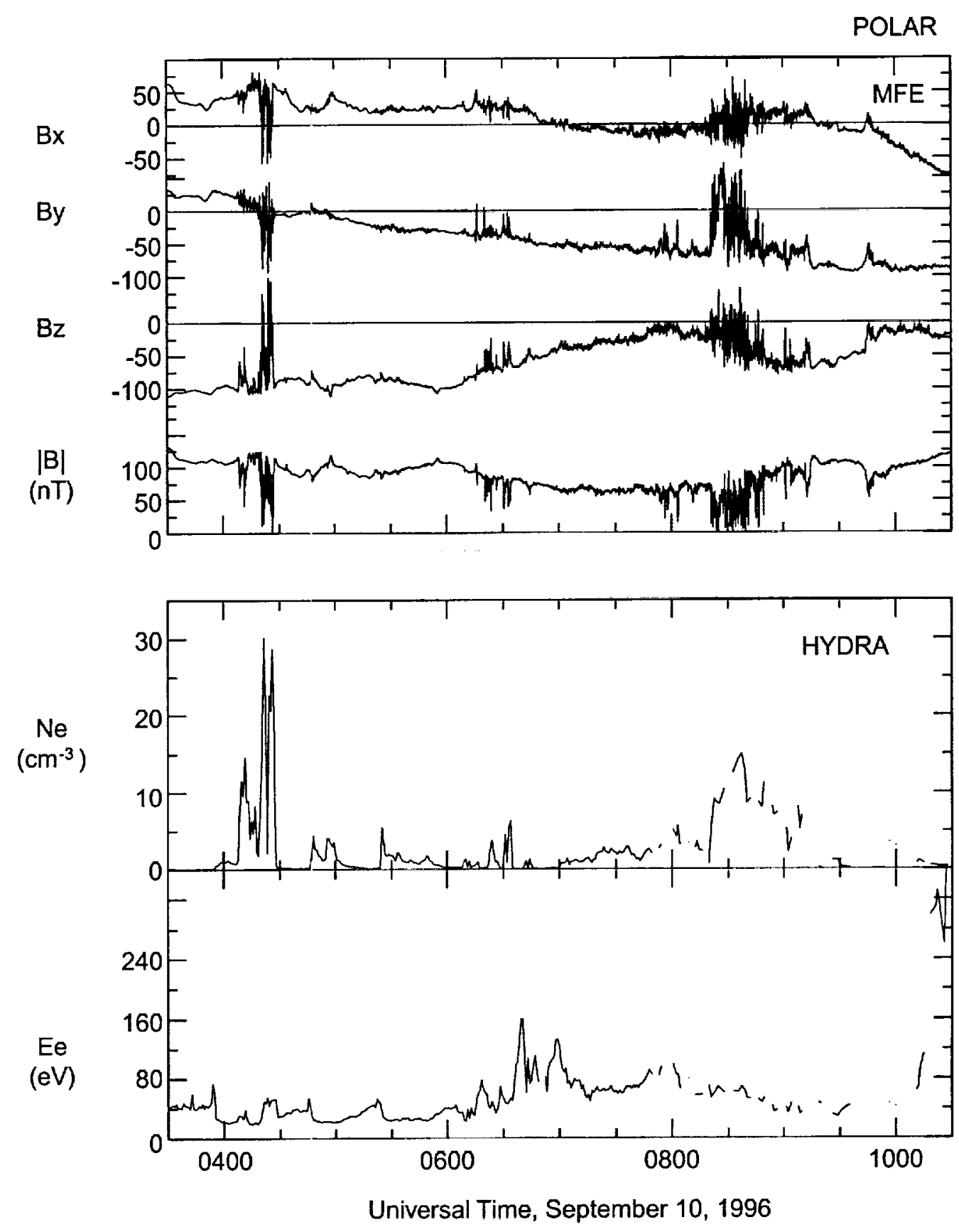

Figure 6. An overview of Polar observations of the cusp region on September 10, 1996. The top panel shows the magnetic field data in SM coordinates. The bottom panel shows Polar Hydra electron density and average energy key parameters. 

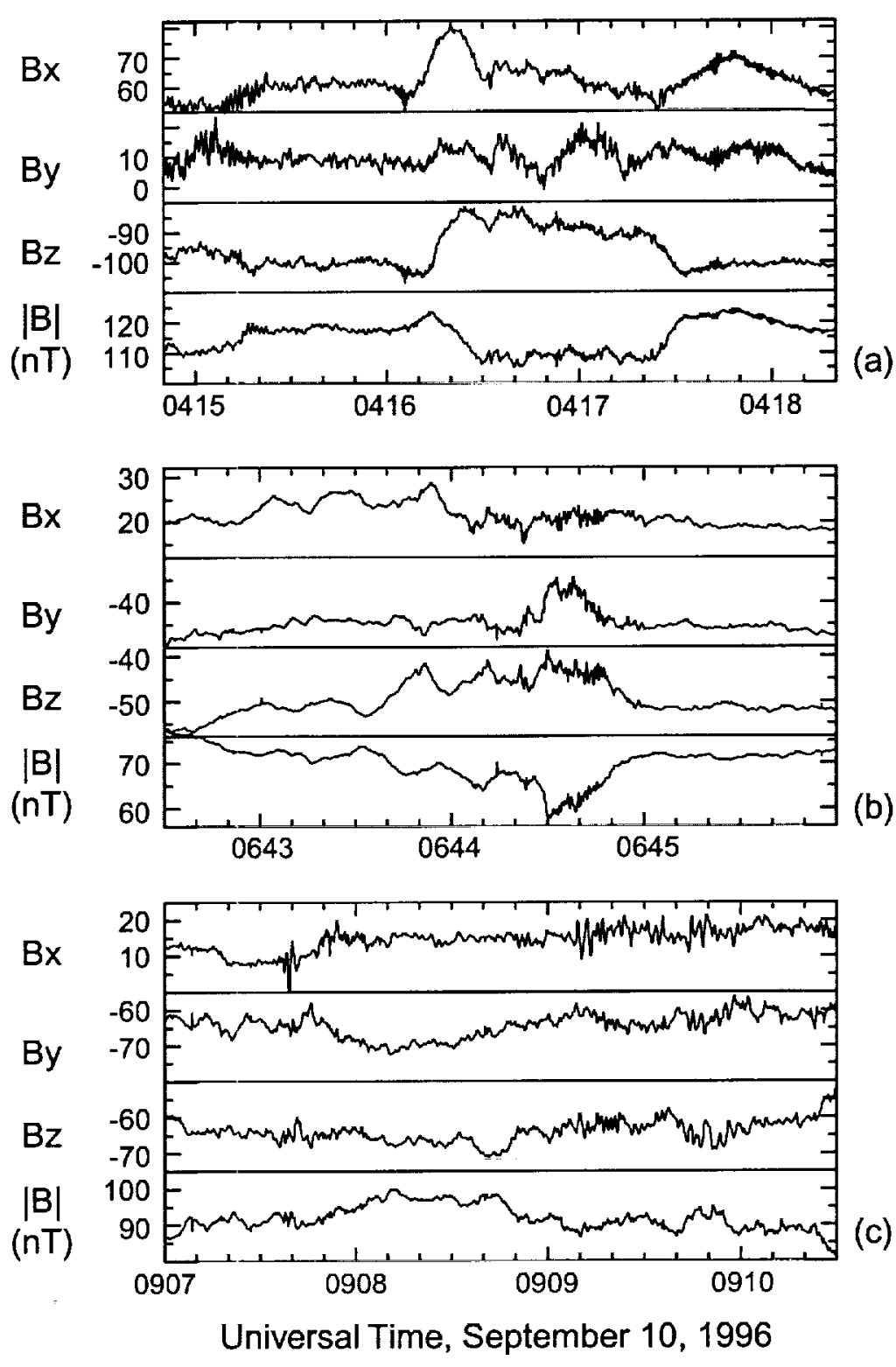

Figure 7. Examples of the waves observed in the field depression region poleward of the cusp (a and b) and in the cusp region (c) on September 10, 1996. 

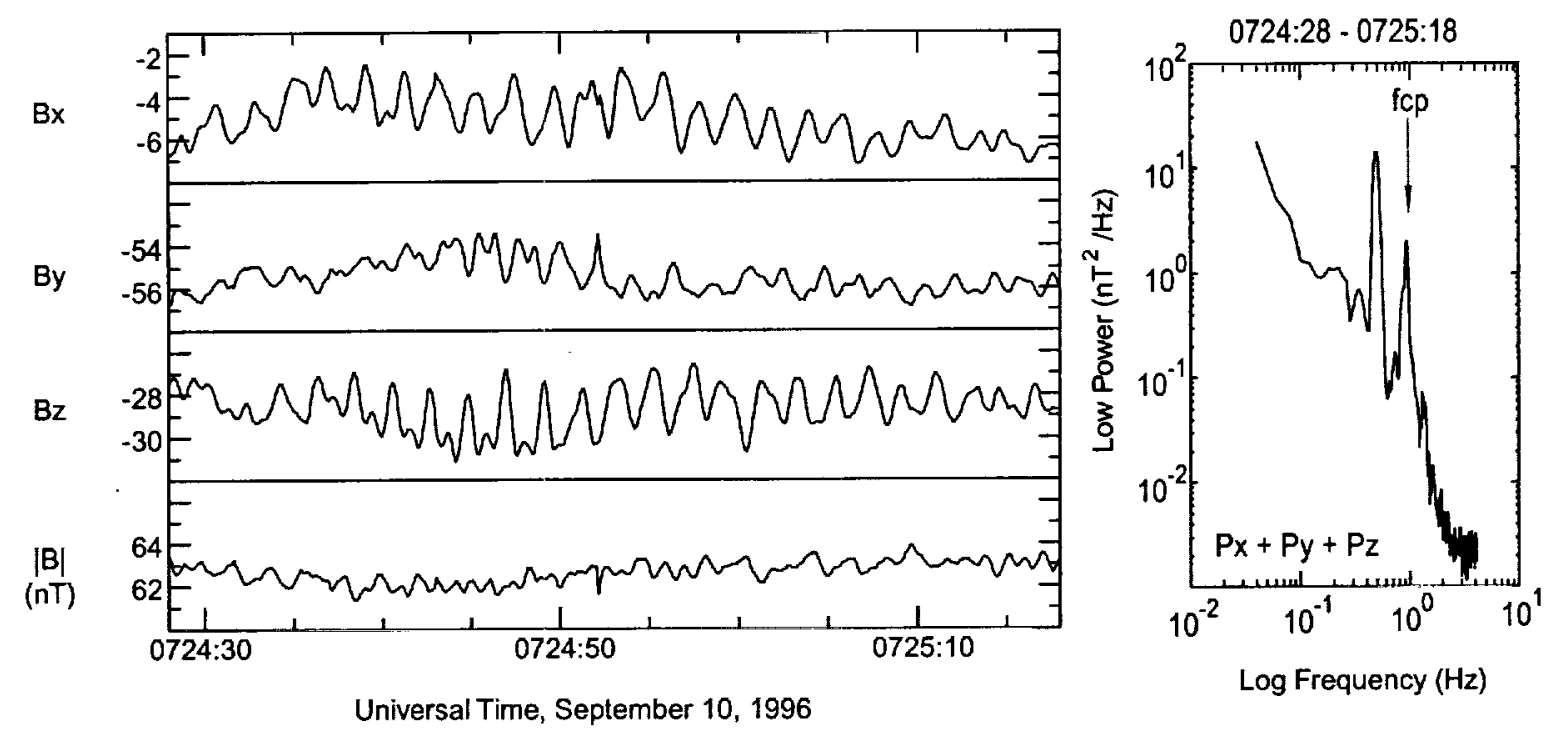

Figure 8. An example of the wave interval with multiple narrow band waves on September 10, 1996. The frequencies of the two spectral peaks are $0.49 \mathrm{~Hz}(0.51 \mathrm{fcp})$ and $0.91 \mathrm{~Hz}(0.96 \mathrm{fcp})$, respectively. 

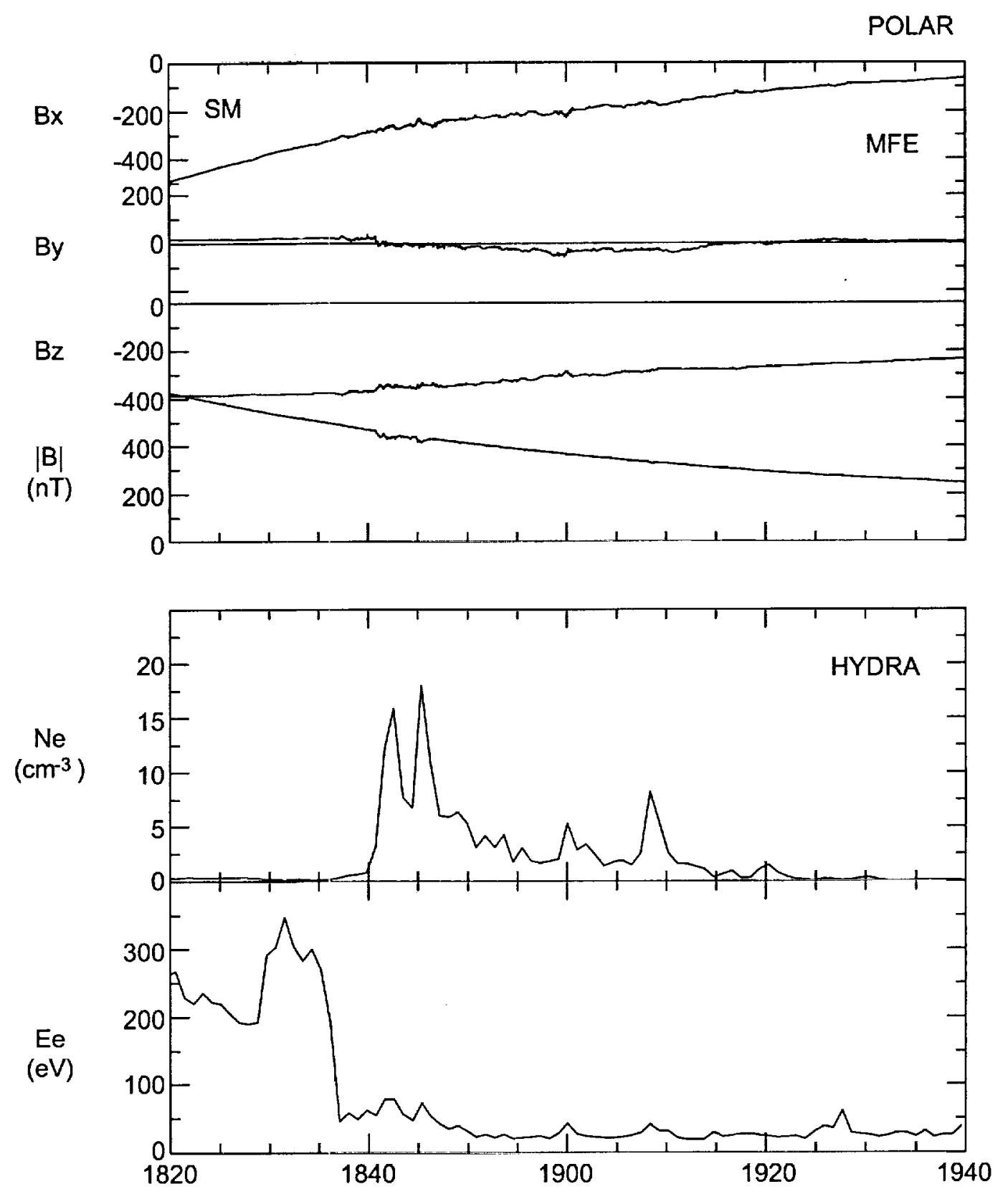

Universal Time, April 21, 1996

Figure 9. An overview of Polar observations during the April 21, 1996 cusp crossing, one of the lowest-altitude crossings in the Polar data surveyed. The top panel shows the magnetic field data in SM coordinates. The bottom panel shows Polar Hydra electron density and average energy key parameters. 


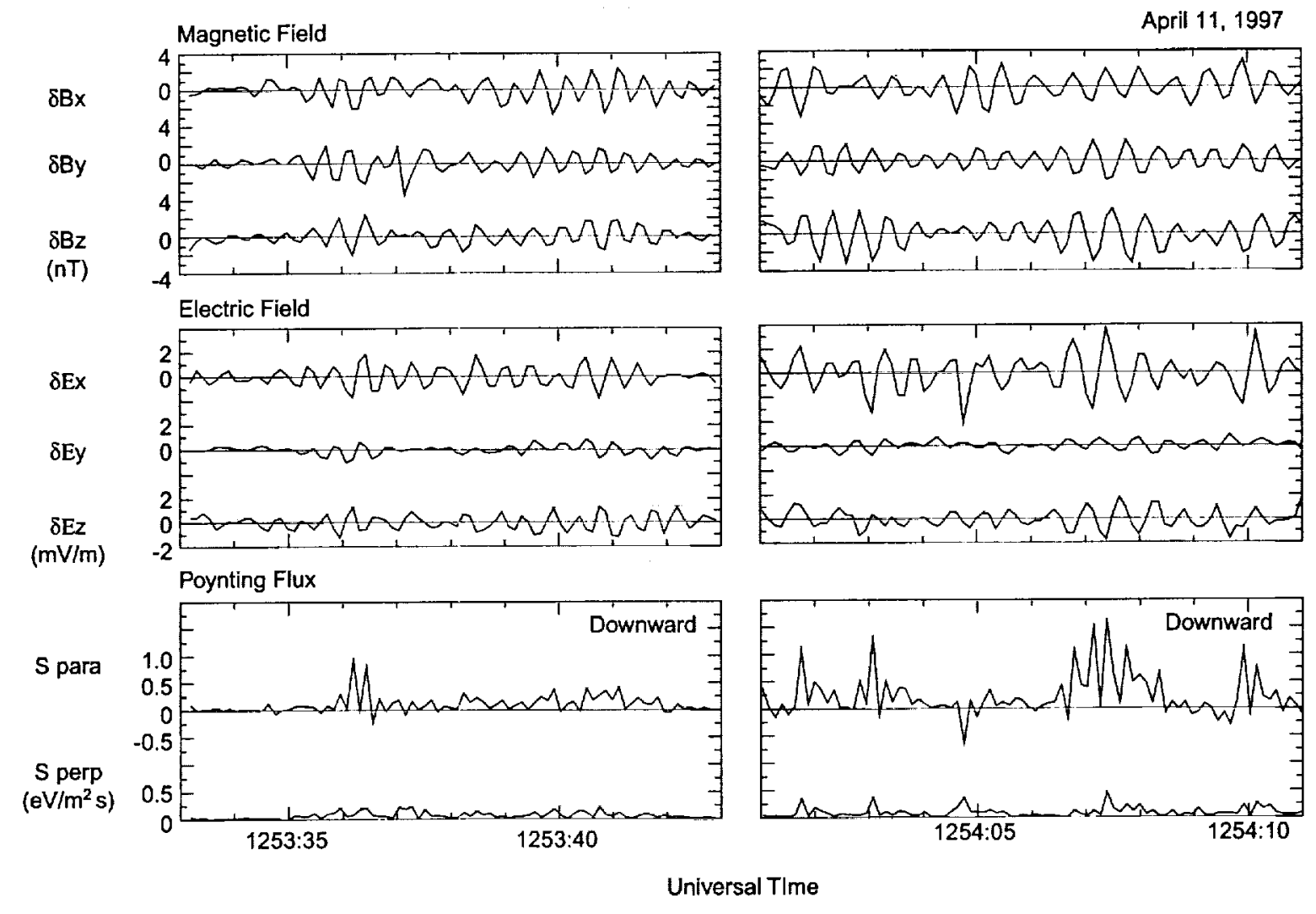

Figure 11. Hi-pass filtered magnetic field, electric field, and Poynting flux for two wave intervals during the April 11, 1997 cusp crossing. 

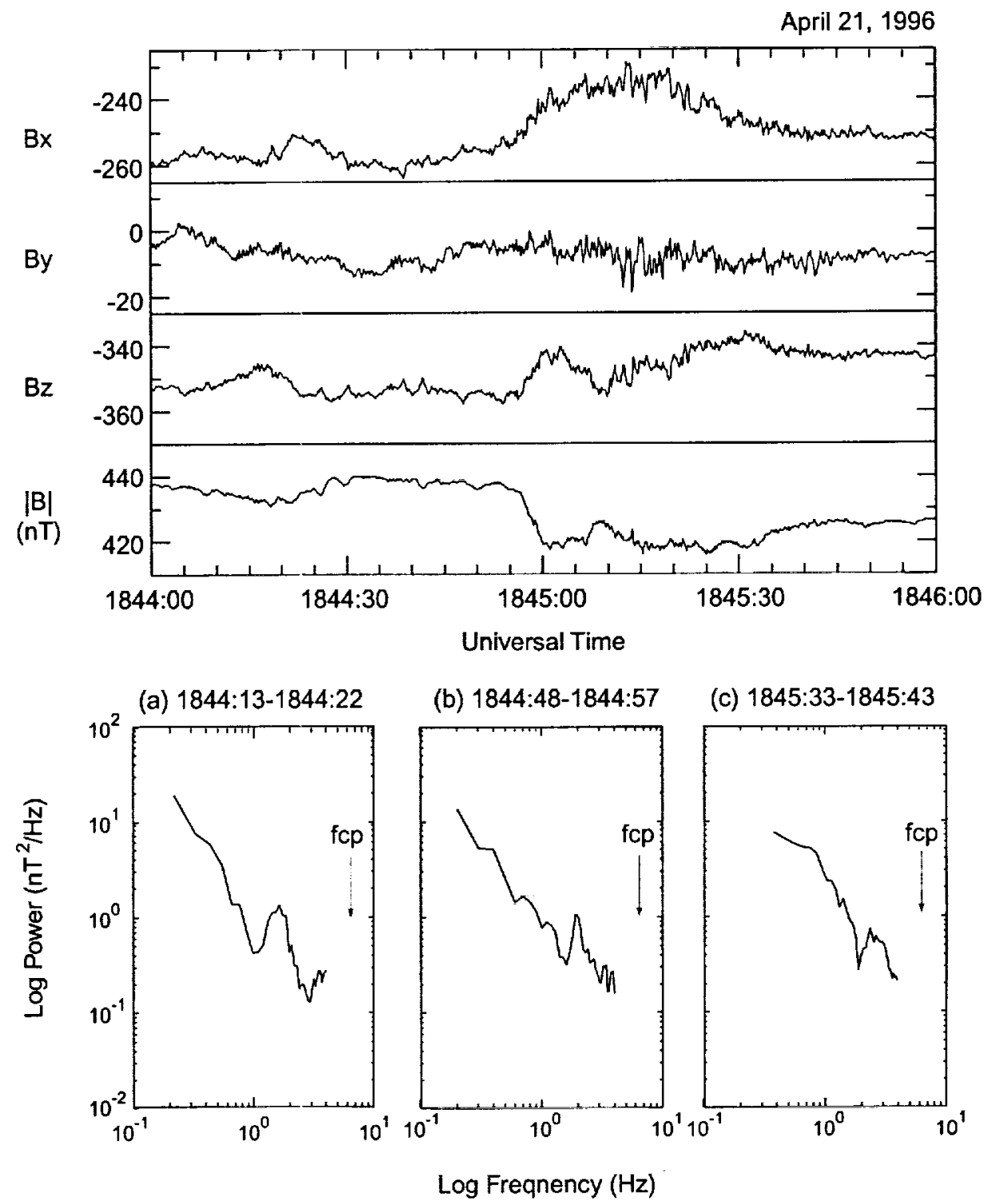

Figure 10. An example of the waves observed during the April 21, 1996 cusp crossing, one of the lowest-altitude crossings in the Polar data surveyed. The bottom panels are the power spectra for three wave packets. The frequencies of the spectral peaks are (a) $1.59 \mathrm{~Hz}(0.24 \mathrm{fcp})$, (b) $2.05 \mathrm{~Hz}$ $(0.31 \mathrm{fcp})$, and (c) $2.56 \mathrm{~Hz}(0.40 \mathrm{fcp})$. 


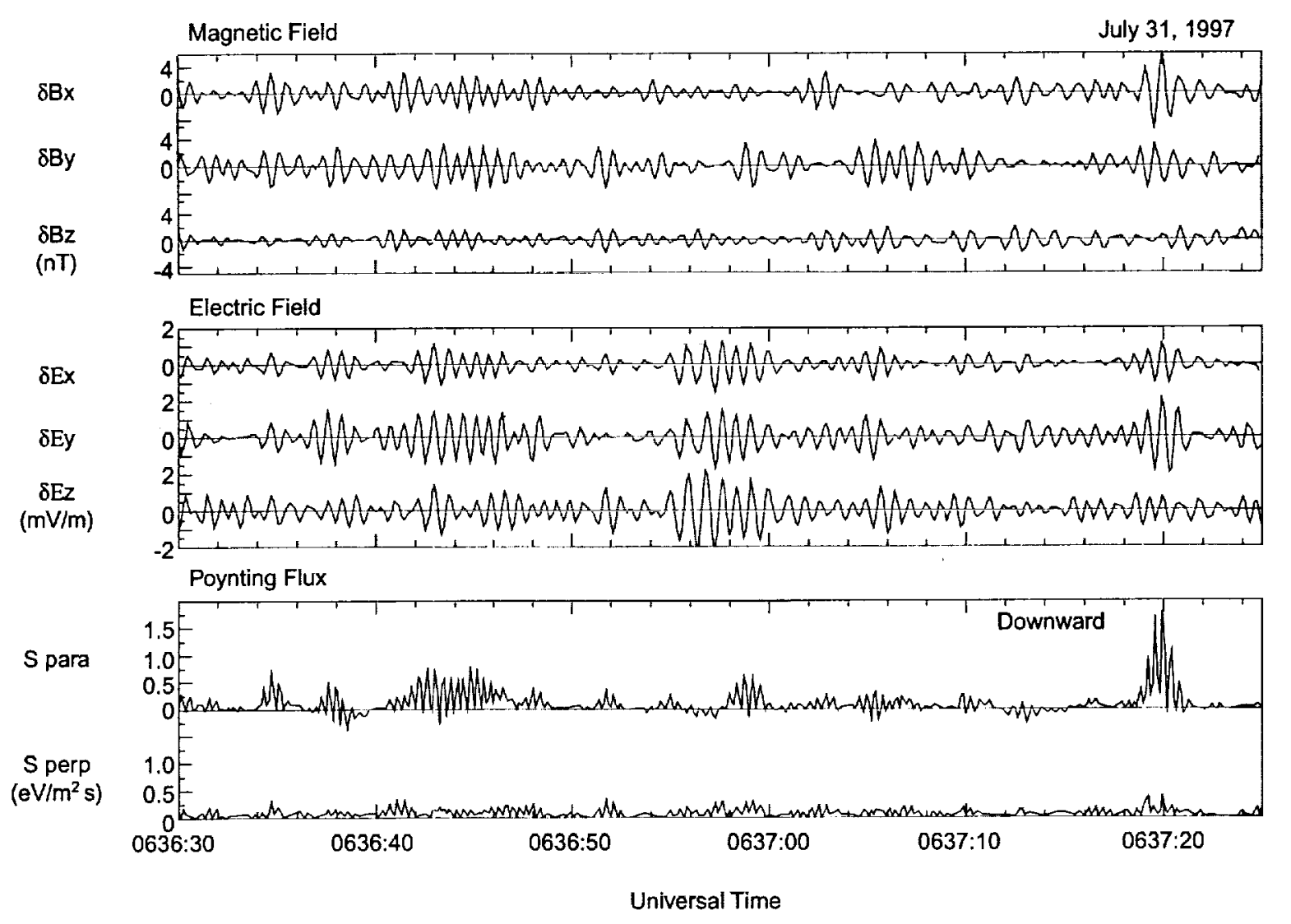

Figure 12. Hi-pass filtered magnetic field, electric field, and Poynting flux for a wave interval during the July 31,1997 cusp crossing. 